\title{
Baggage Carousel Assignment at Airports: Model and Case Study
}

\author{
Barth, Torben C.; Pisinger, David
}

\section{Published in:}

SN Operations Research Forum

Link to article, DOI:

10.1007/s43069-020-00040-1

Publication date:

2021

Document Version

Peer reviewed version

Link back to DTU Orbit

Citation (APA):

Barth, T. C., \& Pisinger, D. (2021). Baggage Carousel Assignment at Airports: Model and Case Study. SN Operations Research Forum, 2(5). https://doi.org/10.1007/s43069-020-00040-1

\section{General rights}

Copyright and moral rights for the publications made accessible in the public portal are retained by the authors and/or other copyright owners and it is a condition of accessing publications that users recognise and abide by the legal requirements associated with these rights.

- Users may download and print one copy of any publication from the public portal for the purpose of private study or research.

- You may not further distribute the material or use it for any profit-making activity or commercial gain

- You may freely distribute the URL identifying the publication in the public portal

If you believe that this document breaches copyright please contact us providing details, and we will remove access to the work immediately and investigate your claim 


\title{
Baggage Carousel Assignment at Airports: Model and Case Study
}

\author{
December 11, 2020 \\ Torben C. Barth ${ }^{1,2}$ David Pisinger ${ }^{2}$ \\ ${ }^{1}$ Information and Telecommunication Services, Fraport $A G$ \\ ${ }^{2}$ DTU Management, Technical University of Denmark
}

\begin{abstract}
Baggage operations at an airport are split into several different sub problems. The problem of assigning baggage carousels to each arriving flight is part of the important passenger arrival process. This article presents a mixed-integer problem for the underlying decision problem which aims at balancing customer satisfaction with operational needs. We introduce a static model that fulfills the different real-world requirements and balances the different objective criteria. It is shown how the model can be used in the dynamic environment of an airport. Because of the high degree of uncertainty at an airport, the issue of stability is discussed in detail. In a case study several scenarios with different time horizons and times of decision were studied. The model was verified with data from Frankfurt Airport and is successfully running as part of the decision support system at Frankfurt Airport.
\end{abstract}

Keywords: Airport baggage handling, Mixed-integer problem, Real-world optimization, Decision support 


\section{Introduction}

Baggage handling is one of the most important tasks during the ground time of an aircraft. For each aircraft arriving at and departing from an airport many decisions related to baggage handling have to be made. Since baggage claim is one of the last touch points for the passenger at an airport the performance at the baggage claim is critical for the airport's success.

The problem investigated in this article is the assignment of a baggage carousel to every arriving aircraft during the daily airport operations. This process has large impact on passenger's satisfaction since passengers directly perceive baggage handling quality. Examples of such situations are crowded baggage carousels, prolonged waiting times or long distances to the baggage carousel. A study by SITA [19] shows that the positive emotions of passengers drop to below $54 \%$ if waiting time at reclaim belts is exceeding 30 minutes. If the waiting time is below 10 minutes, the positive emotions of passengers are $88 \%$. Furthermore, the different needs of airlines, handling companies and the airport operator have to be fulfilled. We name the problem for assigning baggage carousels to arriving flights the baggage carousel assignment problem (BCAP). In this article we propose a mixed integer formulation with a multi criteria objective. The multi criteria objective considers the different stake-holders and operational needs e.g. stability of plans.

In the past literature, baggage claim was mainly considered on a strategic level. The design of baggage claim was analyzed as part of the airport system. One example is the simple landside aggregate model (SLAM) [3]. Ghobrial et al. [12] described a model to predict performance of baggage claim. Tosic [21] described the baggage claim process as part of airport passenger terminal operations. We observe that the control of daily operations gets more attention in the latest years. Recently, several approaches for the BCAP were presented, see for example [8], [14], [11] and [22]. The approaches differ in the considered setup, planning horizon and the objectives. Especially, the considered objectives are limited and do not cover the situation observed at Frankfurt Airport. In this article we present an approach that is generally suited for real-world application at large hub airports.

Wang et al. [22] presented the first approach for BCAP which solves the problem using reinforcement learning. Based on historic assignment decisions the algorithm learns to find good assignments. The approach has the advantage that it can include uncertainties by design. On the contrary, changes in the processes or the emergence of new resources cannot be handled by the algorithm. Furthermore, explicit changes in the decision strategy cannot be included.

Inbound baggage handling was also studied as part of simulation studies $[13,15]$. Huang et al. [13] focused on robustness in their study. They examine if a robust schedule for a whole day can be calculated with only a small amount of rescheduling during the day. The study does not include any other objective criteria. Malandri et al. [15] propose a discrete-event simulation to identify bottlenecks in inbound baggage handling operations.

Pisinger and Scatamacchia [18] study the inbound baggage handling where the main objective is to match the time of unloading to the expected arrival time of the passengers in order to avoid congestion problems in Copenhagen Airport. A decomposition algorithm is presented in which the master problem ensures that each flight is handled, while the subproblem handles timing and overlap issues. Pisinger and Rude [17] report results for inbound baggage handling 
in Copenhagen airport, but do not present algorithmic details.

Related problems include the process for handling bags of departing aircraft (outbound luggage handling) and from passengers changing aircraft at an airport (transfer baggage problem). These problems can be tackled by similar methods and models. The models differ mainly in the considered constraints and the objective functions. The transfer baggage problem was studied by Barth and Franz [6]. Work on the handling of outbound luggage handling was presented by Abdelghany et al. [1], Frey et al. [10], Asco et al. [4], Barth and Pisinger [7] and Pisinger and Rude [17].

The BCAP is a special type of the assignment problem (AP). Classifying the BCAP according to Pentico's survey of APs [16], the BCAP has properties covering several variants of the AP. The most important variant is the generalized assignment problem (allowing more than one flight per baggage carousel). The BCAP can also be seen as a multiple resource general assignment problem (if additional resources are included in the BCAP like space in passenger waiting area) and as a multi-period assignment problem (several flights can be planned consecutively to a baggage carousel without violating the capacity restriction). Furthermore, we have different objective criteria that, e.g., aim at balancing the workload, or balance the use of baggage claim halls and carousels. These balancing criteria can be formulated similar to the bottleneck assignment problem, the balanced assignment problem or the minimum deviation assignment problem. Since none of the existing models covers all constraints and objectives of the described BCAP, we decided to develop a new specific model for the BCAP. This model combines and extends the presented models from Pentico's survey, with the major difference that we consider all versions of capacity constraints as soft constraints. Including the soft constraints in the objective makes the model similar to weighted goal programming approaches. A description of different goal programming approaches can be found in Tamiz [20].

The contribution of the presented model is to introduce a general model which can be applied to different airport setups considering all challenges when applying the model to make decisions in a real world setting. The model is overcoming weaknesses of existing solution approaches which either neglect important operational requirements or are focused to only one specific airport setup. In addition to the obvious benefits of an improved and more stable planning in the daily operations, the model was also applied to support strategic decisions by studying important decision parameters. The findings of the case study can help airports and other comparable operational processes to adapt better planning and dispatching strategies.

The remaining paper is structured as follows: In Section 2 we give a detailed problem description and elaborate on the most important requirements for a real-world implementation. Section 3 introduces a static model for the presented problem. Further, we discuss important goals for the modeling, the implementation and possible extensions of the model. Additionally, the aspect of robustness is examined for the introduced model in a dynamic environment. As a next step, in Section 4, we verify the model in a case study. The case study also examines critical factors for the decisions and gives important insights in decision process in airport operations. 


\section{Problem description}

We consider the following setup for the BCAP as seen in Frankfurt Airport: The baggage infrastructure department is responsible for the allocation of baggage carousels to flights. There are several hard constraints limiting the freedom of choice given by the physical infrastructure and the regulations from the authorities e.g. the need of custom controls or passport checks. After applying these constraints for each flight, there exist several assignment possibilities. Before the decision support system was implemented, a human dispatcher decided which flight are assigned to which baggage carousel. The dispatcher was supported by different IT-systems, in which he/she can access different kinds of information and the relevant data are visualized to support the decisions. The final decision needs to be reached before the handling of the bags of the arriving aircraft starts and the passengers arrive in the baggage claim hall.

The handling process can be described as follows: The bags will be unloaded from the aircraft and then transported to the handling facilities at the terminal building depending on the selected baggage carousel. Large flights often require more than one transport trip. In this case the bags will be transported in subsequent trips to the same handling facility. One or more handling facilities belong to each baggage claim hall. We assume that for each baggage carousel there is one infeed station in a handling facility and that these infeed stations are directly connected to baggage carousels e.g. by conveyor belts. The bags will be unloaded to the infeed stations and then delivered by conveyor belts to the baggage carousels in the baggage claim hall. Finally, the passengers collect their bags at the baggage carousel. The process is summarized in Figure 1. The assignment of a baggage carousel to a flight by the dispatcher has substantial influence on each process step except the unloading of the bags. The assignment decision defines processing times and can cause capacity conflicts. Therefore the decision is crucial for the process efficiency (effort for transportation and handling at infeed stations) and quality (passenger experience). An example for a possible layout at an airport is shown in Figure 2.

We assume that the scheduling of staff for the unloading from the aircraft as well as the scheduling of the transports are separate problems which are solved independently from the BCAP. The duration of these processes can be calculated based on past experiences. The current staff availability in the handling facilities is defined in the given data sets.

\subsection{Problem features of the real-world problem}

When solving the presented problem in a real-world environment, there are different challenges to take into consideration. This section presents the most important features of the problem.

The number of available handling resources is limited, and decisions should thus ensure these resources are utilized in a beneficial way, fulfilling the objectives to the best possible degree. Our experiences at airports show that it is important to incorporate the following features:

(p1) Airline preferences: In some cases the airlines prefer specific baggage carousels e.g. in spatial proximity to the handling agent for missing bags or the same baggage carousel for all flights from the same city. 


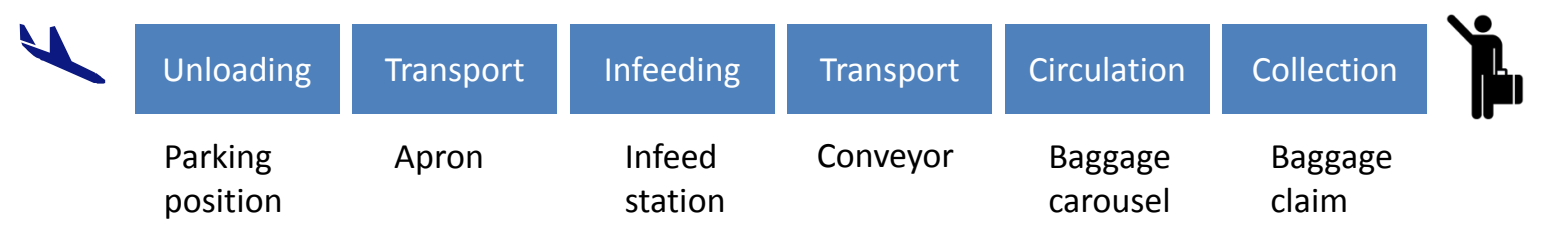

Figure 1: Process steps of inbound baggage handling at an airport.

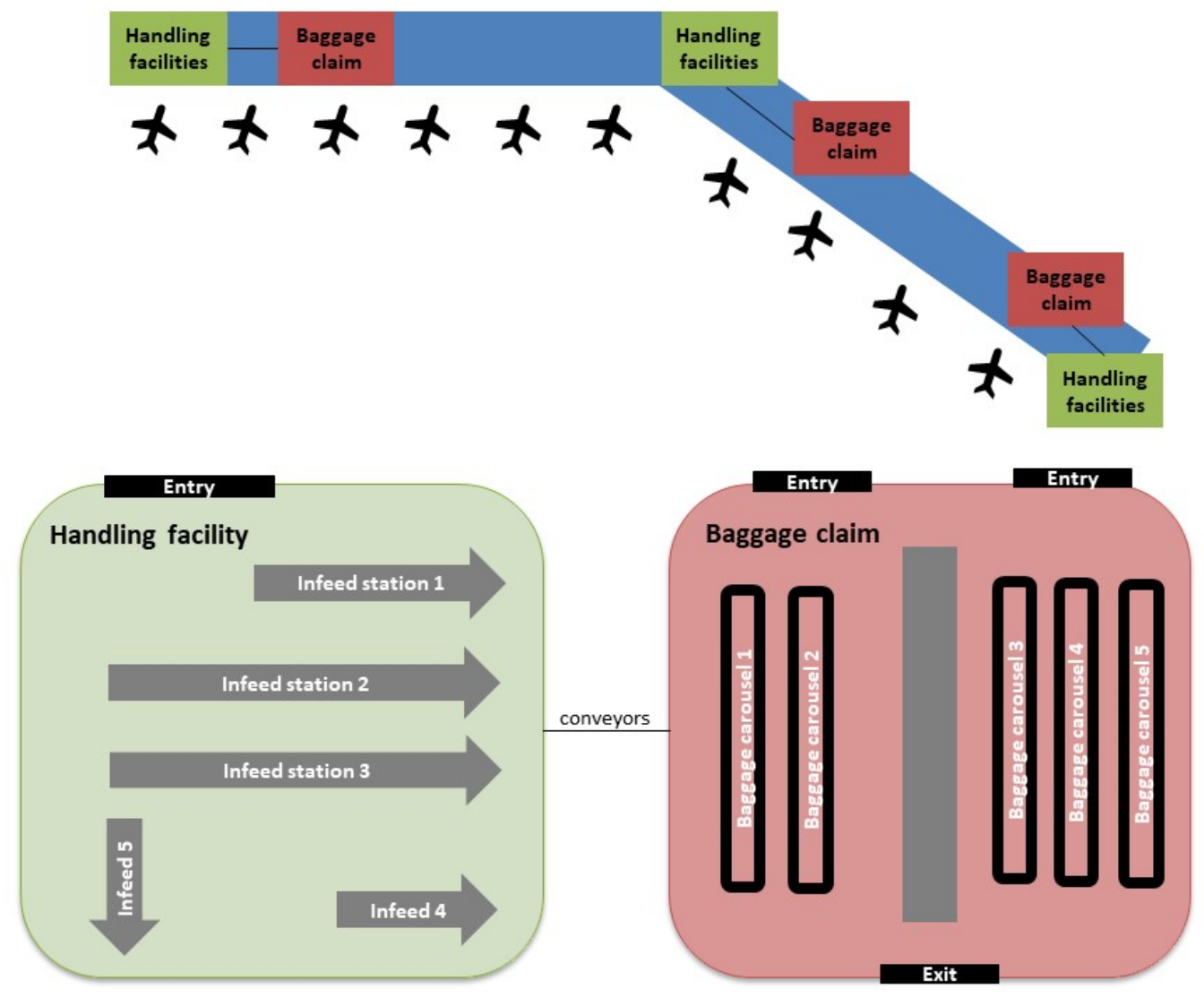

Figure 2: The figure illustrates the layout of the different locations in the process. The upper half shows parking positions, handling facilities and baggage claim. The lower half shows a detailed view of a handling facility and a baggage claim area. The baggage claim area is connected by conveyor belts with the handling facility. 
(p2) Transportation time: The distance from the aircraft parking position to handling facilities should be short so that the bags are available as fast as possible for the passengers and the operator of transport can work efficiently.

(p3) Operational suitability of baggage carousels: The baggage carousels differ in general in the suitability to handle flights e.g. accessibility of baggage carousel with baggage tractors or the accessibility for the passengers. More accessible carousels should be preferred over less accessible.

(p4) Operational suitability of baggage carousels for a specific flight: The baggage carousels can have different capacities to handle specific flights in terms of number of passengers, bags, containers or palettes. For example some baggage carousels are not suited to handle flights with palettes.

(p5) Avoidance of simultaneous handling: Two flights should not be handled simultaneously at one baggage carousel. The avoidance of simultaneous handling of large flights (with respect to the number of bags) is even more important.

(p6) Avoidance of simultaneous handling at neighboring carousels: The simultaneous handling of two flights with many passengers at neighboring baggage carousels should be avoided since the space for passengers is limited.

(p7) Capacity buffer: Since delays or early deliveries should not cause immediate handling problems a buffer between the handling of two flights should be planned.

(p8) Capacity limit in baggage claim halls: The number of flights handled simultaneously in a baggage claim hall is limited by the number of available workers.

(p9) Display limitations: At some airports the number of flights which can simultaneously be displayed at a baggage carousel is limited. It should be avoided to assign more flights than the capacity of the display.

(p10) Balanced utilization of carousels in baggage claim halls: In order to ensure an efficient resource utilization, a balanced distribution over all baggage carousels is desired for each baggage claim hall. For example it is aimed for a balanced workload for achieving synchronized maintenance intervals. In particular that could mean the same amount of bags or flights in a certain period of time.

(p11) Balanced utilization of all baggage claim halls: For efficient resource utilization a balanced distribution over all baggage claim halls is desired. Furthermore, the workload should be distributed equally amongst all workers.

(p12) Forbidden baggage carousels for specific flights: The physical infrastructure limits the choice of baggage carousels for specific flights since it is not possible to walk from every gate to every baggage claim hall. Furthermore, restrictions can be imposed by border controls and customs. 
Table 1: Examples of uncertainty: Average number of position and time changes during the defined time period before the arrival of the aircraft

\begin{tabular}{lrrr}
\hline Minutes before arrival of flight & 60 & 30 & 10 \\
\hline Position changes per flight & 4.92 & 0.3 & 0.02 \\
Time updates per flight & 7.22 & 4.12 & 0.57 \\
\hline
\end{tabular}

This list of important features for solving the BCAP is caused by the different interests of the stake-holders. Features (p1) and (p2) are concerned with customer satisfaction. Features (p2)-(p4) cover operational efficiency and features (p5)-(p12) capacity constraints. Normally, the airport authorities are interested in satisfied customers (passengers and airlines) and in efficient processes (operational needs and constraints of ground handling companies and the airport infrastructure) and seek to combine the different aspects. Some of the described goals depend on the infrastructure of the specific airport.

In this paper we consider the case of an inhomogeneous set of baggage carousels. This is common for hub airports which have been extended several times in the past. In the homogeneous case some of the listed features e.g. suitability for the handling of a specific flight or transportation times can be neglected. For more details on the specific setup see [2] and [5].

\subsection{Uncertainty}

Another important aspect is how to handle the high degree of uncertainty at an airport. When the final assignment decision has to be made, some data is still unknown and influence the solution quality. Examples of uncertain data are:

- The exact final arrival time of the aircraft

- The final aircraft parking position (the planned position can change on short notice)

- The exact duration for unloading depends on the loading order and the availability of staff

- The exact transportation time from the aircraft to the handling facilities can vary for each trip since it depends on punctual arrival of the driver and the amount of apron traffic during the trip

- The exact number of bags transported on each trip is often unknown

Table 1 shows how uncertainty of the parking position and arrival time decreases until the arrival of the aircraft at the parking position. On average each flight has 5 position changes, and 7 updates of the arrival time in the last hour before arrival, making it hard to predict the exact point in time when the resources will be occupied.

This high degree of uncertainty is the reason why we discuss the issue of robustness of the generated assignments and the appropriate timing for the decision for each flight in the following 
sections in more detail. An important decision is to decide the resolution of the model. In our opinion a high resolution (e.g. calculation on single minutes) - as observed in similar approaches - can be impracticable since it is hard to predict the arrival of the bags accurately.

In order to measure the quality of our algorithms and different decision strategies we introduce a quality parameter $q$. The parameter $q$ is defined as the ratio between the objective obtained by an algorithm or specific decision strategy, and the best possible solution obtainable if all data is known in advance. The global best solutions is hence determined under the knowledge of e.g. the final parking position, and the real arrival time of each flight. To calculate $q$ we extract all results produced by an algorithm or specific decision strategy and solve the optimization model again for a whole day with all flights fixed to the extracted results. The global best solution is determined by solving the model for all flights of the day at once under the knowledge of all data. A quality $q$ of 1.0 thus means that the objective of the algorithm or decision strategy is equal to the global best solution.

\section{Model}

Section 2 introduced the problem and the features that need to be satisfied in a real-world implementation. In this section we present a model which handles all the described features and can be used as decision support. All parameters and variables are defined in the text, but for ease of reference, an overview can be found in Appendix A, Tables 6-8.

\subsection{Static model}

The BCAP can be modeled as an extended assignment problem. The solution of the problem consists of an assignment of each flight to one baggage carousel.

Given a set of flights $F$ and a set of baggage carousels $B$. Each baggage carousel $b \in B$ belongs to one baggage claim hall $h$ in the set of all baggage claim halls $H$. The set of all baggage carousels in one baggage claim hall $h$ is denoted $B_{h}$. For each flight $f \in F$ a feasible assignment which optimizes the given goals has to be selected.

It is possible to precalculate all legal assignments, since the solution space is limited by the available baggage carousels. The set of all assignments is denoted as $A$ and the set of all legal assignments for a specific flight $f$ as $A_{f}$. We assume that all hard constraints restricting the choice of baggage carousels are respected when generating the set $A_{f}$ for each flight $f \in F$ (see feature (p12) from the list in Section 2.1).

The model considers only flights in a specific time horizon. For calculating the resource consumption, it is necessary to split the time horizon into equisized timeslots. The set of all timeslots during the considered time horizon is denoted by $T$. The length of one timeslot $t \in T$ is $l$ and the planning horizon is thus given by $L=|T| \cdot l$. We assume that we only include flights which arrive in the considered time horizon and can be handled within the time horizon.

The major decision variable of the model is $x_{a}$ for each $a \in A$. This variable indicates if an assignment is chosen or not. Furthermore, some auxiliary variables $y \geq 0$ are used to calculate 
the violation of soft constraints. The $y$ variables are a consequence of the assignments $x_{a}$ and hence only the latter are actual decision variables.

The main constraints of our model are the assignment constraints:

$$
\begin{aligned}
& \sum_{a \in A_{f}} x_{a}=1 \quad \forall f \in F \\
& x_{a} \in\{0,1\} \quad \forall a \in A
\end{aligned}
$$

Equation (1) ensures that exactly one assignment is selected for each flight. Feature (p12) is clearly a hard constraint which must not be violated. This constraint is applied during the generation of the assignments out of performance reasons and therefore is not included explicitly.

All further constraints are soft constraints. Each soft constraint $s$ is defined for a set of resources $r \in R_{s}$. The use of resource $r$ in timeslot $t$ by assignment $a$ is denoted as $u_{a, r, t}$.

The proposed model uses the concept of soft constraints for two reasons. One reason is the degree of uncertainty when the decision is made (see Section 2.2). Since it is not possible to predict the exact arrival times and the order of the arriving trips of the different flights it does not make sense to insist on hard constraints. Secondly, soft constraints express the real process in an appropriate manner. Physically, it is not possible to simultaneously handle bags from two flights at the same carousel, but in reality the bags will be handled sequentially according to the arrival order. The solution is always feasible but a simultaneous assignment can mean that a waiting time for one of the flights occurs. This situation should ideally not appear but will be tolerated during peak hours.

The first group of soft constraints is concerned with the capacities of the defined resources. Set $S$ contains soft constraints $s$ which can be individually defined for each airport. $C A P_{r, t}$ defines the available capacity of resource $r \in R$ at each time slot $t$ which should not be exceeded. The variable $y_{r, t}$ measures the violation of $C A P_{r, t}$.

$$
\sum_{a \in A} u_{a, r, t} \cdot x_{a} \leq C A P_{r, t}+y_{r, t} \quad \forall r \in R_{s}, \forall t \in T
$$

This soft constraint type is defined for all resources listed in Table 2 at Frankfurt Airport. For each resource we describe the set of resources $R_{s}$ and the capacity $C A P_{r, t}$.

Constraints (c2)-(c5) from the table aim to avoid simultaneous handling from more than one flight at a baggage carousel (feature (p5) from Section 2.1). During peak hours it might be necessary for more than one flight to share the same baggage carousel, therefore we decided to introduce a stepwise approach. The penalty of the violation increases with a higher number of flights handled simultaneously. Resource consumption $u_{a, r, t}$ is defined on the basis of the number of waiting passengers at a baggage carousel at a specific time slot for constraint (c1) (feature (p6)). For constraints (c2)-(c5) resource consumption $u_{a, r, t}$ is one for all time slots $t$ between the handling of the first and last bag of flight $f$ at the baggage carousel belonging to assignment $a$. Resource consumption is defined analogously on baggage claim halls (feature (p8)) for constraint (c6). For constraint (c7) resource consumption is one during the display time of a flight which depends on the specific rules of the airport (feature (p9)). For instance, a flight 
Table 2: This table shows all resources modeled by a capacity soft constraint (17). The capacity constraints correspond to features (p5)-(p9) in the model.

\begin{tabular}{|c|c|c|c|}
\hline & Description of soft constraint $s$ & Set of resource $R_{S}$ & Capacity $C A P_{r, t}$ \\
\hline (c1) & $\begin{array}{l}\text { Limit of simultaneous passengers at } \\
\text { neighboring baggage carousels }\end{array}$ & $\begin{array}{l}\text { All pairs of baggage } \\
\text { carousels }\end{array}$ & $\begin{array}{l}\text { Specific passenger capacity } \\
\text { per pair }\end{array}$ \\
\hline (c2) & Simultaneous handling of one flight & All baggage carousels & One flight per timeslot \\
\hline (c3) & $\begin{array}{l}\text { Simultaneous handling of two } \\
\text { flights }\end{array}$ & All baggage carousels & Two flights per tiı \\
\hline (c4) & $\begin{array}{l}\text { Simultaneous handling of three } \\
\text { flights }\end{array}$ & All baggage carousels & Three flight per timeslot \\
\hline$(\mathrm{c} 5)$ & $\begin{array}{l}\text { Simultaneous handling of large } \\
\text { flights }\end{array}$ & All baggage carousels & One large fli \\
\hline (c6) & $\begin{array}{l}\text { Limit of flights in baggage claim } \\
\text { hall }\end{array}$ & All baggage claim halls & $\begin{array}{l}\text { Number of available work- } \\
\text { ers per timeslot }\end{array}$ \\
\hline (c7) & Display limit & All baggage carousels & $\begin{array}{l}\text { Given capacity limit of the } \\
\text { displays }\end{array}$ \\
\hline
\end{tabular}

is only shown on the passenger info displays after arrival, while it may be shown on info displays for the handlers long time before arrival.

The following group of constraints ensures that the baggage claim halls are used in a balanced way (feature (p11)). First, the average usage of all baggage claim halls $y^{B H, m e a n}$ is calculated in equation (4). The positive and negative deviations $y_{h}^{B H,+}$ and $y_{h}^{B H,-}$ from the average is then determined for each baggage claim hall $h$ in equation (5). The absolute deviation $y_{h}^{B H}$ is calculated for each baggage claim hall $h$ in equation (6). In this context the baggage claim halls $h \in H$ are used as resources.

$$
\begin{gathered}
y^{B H, \text { mean }}=\frac{\sum_{a \in A, h \in H, t \in T} u_{a, h, t} \cdot x_{a}}{\sum_{h \in H, t \in T} C A P_{h, t}} \\
y^{B H, \text { mean }}+y_{h}^{B H,+}-y_{h}^{B H,-}=\frac{\sum_{a \in A, t \in T} u_{a, h, t} \cdot x_{a}}{\sum_{t \in T} C A P_{h, t}} \quad \forall h \in H \\
y_{h}^{B H}=y_{h}^{B H,+}+y_{h}^{B H,-} \quad \forall h \in H \\
y^{B H, \text { mean }} \geq 0 \\
y_{h}^{B H} \geq 0, \quad y_{h}^{B H,+} \geq 0, \quad y_{h}^{B H,-} \geq 0 \quad \forall h \in H
\end{gathered}
$$

The next group of constraints is concerned with a balanced utilization of the baggage carousels in each baggage claim hall $h \in H$ (feature (p10)). The distance $y_{h}^{B C}$ between the minimum and maximum usage $y_{h}^{B C, \min }$ and $y_{h}^{B C, \max }$ is calculated in equation (11). Equation (9) calculates the 
Table 3: Direct cost considered in the model for each assignment depending of the assignment of a flight to a baggage carousel corresponding to the features concerned with customer satisfaction and operational efficiency

\begin{tabular}{llll}
\hline & Direct cost $c$ & Type & Feature from Section 2.1 \\
\hline (d1) & Airline preferences & Max & $(\mathrm{p} 1)$ \\
(d2) & Transportation time & Min & $(\mathrm{p} 2)$ \\
(d3) & Suitability of baggage carousels & $\operatorname{Max}$ & $(\mathrm{p} 3)$ \\
(d4) & Suitability for specific flight & $\operatorname{Max}$ & $(\mathrm{p} 4)$ \\
\hline
\end{tabular}

maximal utilization $y_{h}^{B C, \max }$ which needs to be greater than or equal to the utilization of all resources included in the constraint. Equation (10) is defined analogously to equation (9) for the minimum utilization $y_{h}^{B C, \min }$. In this context the baggage carousels $b \in B$ are used as resources.

$$
\begin{gathered}
y_{h}^{B C, \text { max }} \geq \frac{\sum_{a \in A, t \in T} u_{a, b, t} \cdot x_{a}}{\sum_{t \in T} C A P_{b, t}} \quad \forall h \in H, \forall b \in B_{h} \\
y_{h}^{B C, \text { min }} \leq \frac{\sum_{a \in A, t \in T} u_{a, b, t} \cdot x_{a}}{\sum_{t \in T} C A P_{b, t}} \quad \forall h \in H, \forall b \in B_{h} \\
y_{h}^{B C}=y_{h}^{B C, \text { max }}-y_{h}^{B C, \text { min }} \quad \forall h \in H \\
y_{h}^{B C} \geq 0, \quad y_{h}^{B C, \text { max }} \geq 0, \quad y_{h}^{B C, \text { min }} \geq 0 \quad \forall h \in H
\end{gathered}
$$

The objective consists of the direct cost calculated from the selected assignments and the decision variables depending on the soft constraints.

$$
\begin{array}{r}
\min \sum_{a \in A, c \in C} n_{c} \cdot w_{c} \cdot o_{a, c} \cdot x_{a}+\sum_{s \in S} w_{s} \sum_{r \in R_{s}, t \in T} y_{r, t} \\
+w^{B H} \sum_{h \in H} y_{h}^{B H}+w^{B C} \sum_{h \in H} y_{h}^{B C}
\end{array}
$$

The first sum in the objective captures the direct costs, while the next sum corresponds to the soft constraints. The following two sums represent the balancing constraints for the baggage claim hall, resp. the baggage carousels. The priorities of the different cost terms $c$ are expressed by the corresponding weights $w_{c}$. The priorities of the objective terms are given by $w_{s}$ for soft constraint $s$, and for balancing the use of the baggage claim halls by $w^{B H}$ (resp. $w^{B C}$ for balancing the use of carousels). Table 3 lists all direct costs included in the model implemented at Frankfurt Airport. The table states for each cost term if it is a minimization or maximization. For minimization of direct cost term $c$, the parameter $n_{c}$ is set to 1 and for maximization to -1 . For each assignment $a$ and cost term $c$ the cost is given by $o_{a, c}$.

Having multiple - and often conflicting - criteria is quite common for decision making in baggage handling. It was already described in previous work by Barth and Franz [6], Barth 
Table 4: Overview of the priorities of the weights in the implementation at Frankfurt Airport corresponding to features

\begin{tabular}{ll}
\hline Criteria & Priority \\
\hline Airline preferences & high \\
Transportation time & medium \\
Suitability of baggage carousels & low \\
Suitability for specific flight & high \\
Stability bonus (see Section 3.2) & low \\
Limit of simultaneous passengers at neighboring baggage carousels & medium \\
Simultaneous handling of flights & high \\
Violation of buffer constraint (see Section 3.3) & high \\
Limit of flights in baggage claim hall & high \\
Display limit & high \\
Balanced use of baggage claim halls & low \\
Balanced use of baggage carousels & low \\
\hline
\end{tabular}

and Pisinger [7] and Asco et al. [4]. Obviously, the different objective terms are measured in different dimensions e.g. as seconds for the transportation time or the number of flights violating the simultaneous handling restriction. Adjusting the weights according to respect the different dimensions is a part of the needed model calibration when adapting to the setup at a specific airport. In practice, it was found that a separate normalization step for each objective makes a successful implementation more difficult. During the calibration process at a specific airport the priorities of the different objectives will be discussed with the business department. Balancing the different criteria is discussed based on the original not normalized values for each criteria e.g balancing transportation time (measured in seconds) versus the violation of a passenger capacity at neighboring baggage carousels (measured as number of passengers above the defined capacity). A normalization makes it nearly impossible to explain the model calibration to the operational experts and demands an additional translation step.

Table 4 shows how the different criteria were ranked at Frankfurt Airport. The ranking was found through several meetings with stake-holders and management. The weights depend strongly on the setup at a specific airport and the dimension of the input parameters. In Section 3.4 the impact of the different criteria will be discussed.

The model covers all described criteria, besides the uncertainties identified in Section 2.2. The coverage of the uncertainty will be discussed in Section 3.3. All features concerning exclusively the assignment of a single flight to a baggage carousel were converted to direct cost (features (p1)-(p4)). All features (p5)-(p9) depending on the combination of the selected assignments, and concerning different resources, were modeled as soft constraints. The violation of the soft constraints measures the distance to the aspired solution for a specific criteria. For example the aspired goal can be no simultaneous handling of flights or a totally balanced use of the baggage carousels. A detailed explanation concerning the use of the soft constraints will be given in Section 3.4. 
The presented model — including the equations from Section 3.3 - is solvable with a standard MIP solver, which is shown by our results using data from Frankfurt Airport (see Section 4.1).

\subsection{Modeling and implementation issues in a dynamic environment}

The dynamic environment of baggage handling makes it necessary to take a decision for each flight based on the available data. This section describes how the model was applied in a dynamic way at Frankfurt Airport.

In our setup, the model is solved regularly (at least every two minutes) with the most recent data. Each run includes all flights arriving within a fixed time period. The suggestions of a single flight should not change too much between the different runs to increase stability of the schedules. Stability can help in reducing stress caused by changing suggestions of the dispatcher and to improve planning quality of the processes depending on the BCAP e.g. staffing at the handling facilities. To enforce stability we decided to add an additional direct cost term measuring the continuity of the suggestions. This term gives a bonus for an assignment if it was already suggested in the previous run. If the dispatcher has made his final decision the flight is fixed to the chosen baggage carousel. If the model is used in an automated environment the flight will be fixed when the point in time for the final decision has arrived e.g. arrival of the aircraft at the parking position.

The time horizon is moved forward with each run. In order to obtain good results it is necessary to choose the right time horizon of the input data and the model. The time horizon should be chosen in such a way that no resource conflicts between included flights appear after the model's time horizon. It is important to take into account the resource consumption of all flights which are not fully handled and adjust the estimated resource consumption with real values as soon as the real values are known. Choosing the right time horizon for the input data is studied in Section 4.3.

In Section 3.1 a direct cost term measuring the suitability of every single baggage carousel was defined. This suitability combines the suitability for the passengers (easy to reach; short paths; closeness to exit) and the operational needs (accessibility from the apron for the unloading of bags). If each baggage carousel has a unique suitability value, most symmetry disappears from the model since all assignments from one flight will be different and it is furthermore unlikely that two assignments of different flights have the same values (the number of bags or the arrival time will differ in almost all cases). For solving large instances with a MIP solver this property of the model can help speeding up solution time.

For adapting the model to a specific airport, the balancing of the workload in baggage claim halls and between baggage claim halls can be calculated for each time period or for the whole planning interval depending on the needs at the specific setup.

\subsection{Application of the model in a dynamic environment}

As described in Section 2.2 many factors can change during the day of operations in the dynamic environment of an airport. To take into account these fluctuations, and the need to take decisions 
before all data is known, it is necessary to consider uncertainty in the plans. Capacity buffers are introduced to prevent the intersection of handling times of different flights at a baggage carousel because of a change of their arrival times. We decided to introduce additional soft constraints for increasing the quality $q$ of the generated schedules:

$$
\sum_{a \in A} u_{a, b, t, b l} \cdot x_{a} \leq 1+y_{b, t, b l} \quad \forall h \in H, \forall b \in B_{h}, \forall t \in T, \forall b l \in B l
$$

These constraints can be interpreted as buffer constraints. The constraints force the model to plan a buffer time between consecutive flights. It is possible to define different buffer lengths $b l \in B l$. The set of all soft constraints considering the different buffer length $B l$ is a subset of the soft constraints $S$ and therefore part of the objective. For each buffer length $b l$ a violation of the corresponding constraint is counted when more than one flight is handled during the defined buffer time. By introducing different buffer lengths we linearized the constraint. The weights $w_{b l}$ are higher for shorter buffer lengths.

Since it is much more important for operational quality that no overlap of flights with many pieces of bags appear, we decided to add an additional constraint for large flights. This extension of the model should fulfill feature (p7) from list in Section 2.1.

In order to further improve the quality $q$ one can apply a similar approach as applied by Diepen et al. [9] to the gate assignment problem. The idea is to maximize idle time between two consecutive flights. Furthermore, historic arrival profiles (early arrivals and delays) are included to capture the likelihood that a conflict between two flights arises. Applied to our concept of handling uncertainty this means that the optimal buffer time between two flights depends on the historic arrival data of the different flights (the more uncertain arrival times, the larger buffer time). The optimal buffer time can even be different in both directions because the likelihood of early arrival and late arrival is different.

\subsection{Discussion of the model}

All features identified in Section 2.1 are handled by the model and as will be shown in Section 4 the model is solvable even for large instances. All features concerning customer satisfaction and operational efficiency are part of the objective since these features represent the goals of the problem. The features concerning the capacity constraints are part of the (soft) constraints in the presented model.

The solution approach outlined in Section 3.2 for the dynamic use is a heuristic approach based on aggregation of the data. For example the arrival times and the arrival order of the single trips with bags at the handling facilities are not considered. Furthermore, the soft constraints do not exactly model the resource utilization but calculate an average resource utilization for aggregated time intervals. Particularly, the model does not include a flow constraint for modeling the handling capacity on basis of single trips. We decided to use an aggregated approach since not all process details are well predictable and relevant for the decision.

Flexibility is an important point in the airport business since the environment can change several times a year e.g. because of new regulation or security procedures [7]. The presented model is easily extendible with new constraints or objective terms, and the different criteria can 
be adjusted to new situations. Furthermore, the model is independent of the business structure and the infrastructure setup and can be adapted dynamically to different situations e.g. an own set of calibration parameters for situations with resource shortages. Lastly, the solution approach - solving the model with a MIP-solver - does not depend on the specific model.

The impact of the variation of the weights of the different objective criteria was studied in a technical report [5]. Summarized, we have observed that increasing the weights of the balancing criteria makes it harder to solve the problem with a MIP solver. The differences in the objective will become much smaller between the individual assignments and the branch and bound tree needs to be explored in more detail. Furthermore, symmetry elimination helps speeding up the solution time. The introduction of a suitability factor for each baggage carousel eliminates most of the symmetry. In our opinion, this issue does not restrict the model since in practice there is always a preferred baggage carousel for the handling. In general, the structure of the model does not change if additional direct costs or soft constraints are added.

Summarized, we see the following strengths of our model:

- The solution method is not affected by adding new objective criteria (direct cost for single assignments or soft constraints depending on the combination of different flights) which can be the case for a meta-heuristic or a column generation approach

- Evaluation and comparison of solutions based on objectives is easy

- Solutions are reproducible and optimal on basis of the defined model

- Solutions cope with the underlying uncertainty (with the help of the concept of soft constraints) and the model, when applied in the described way, produce good quality values $q$ close to 1.0

- The model ensures feasibility in all situations

- The model can be solved to optimality with a standard MIP solver

Potential drawbacks of the presented model are: The effort for calibrating the model is high. It is necessary to find the appropriate weights for the objective terms. Furthermore, the time intervals need to be defined for the soft constraints. Another issue is the stability of the solutions between different optimization runs since a small time change can affect the whole plan and result in strongly deviating plans compared to the plan of the last run. Finally, the model gives no upper limit on solution time - we did not observe any problem in solution time since the implementation is running at Frankfurt Airport.

\section{Case study}

This section shows the results of a case study based on the presented model. The aim of the case study was to verify the model, to show that the model helps improving daily operations and to study important decision parameters of the BCAP. 


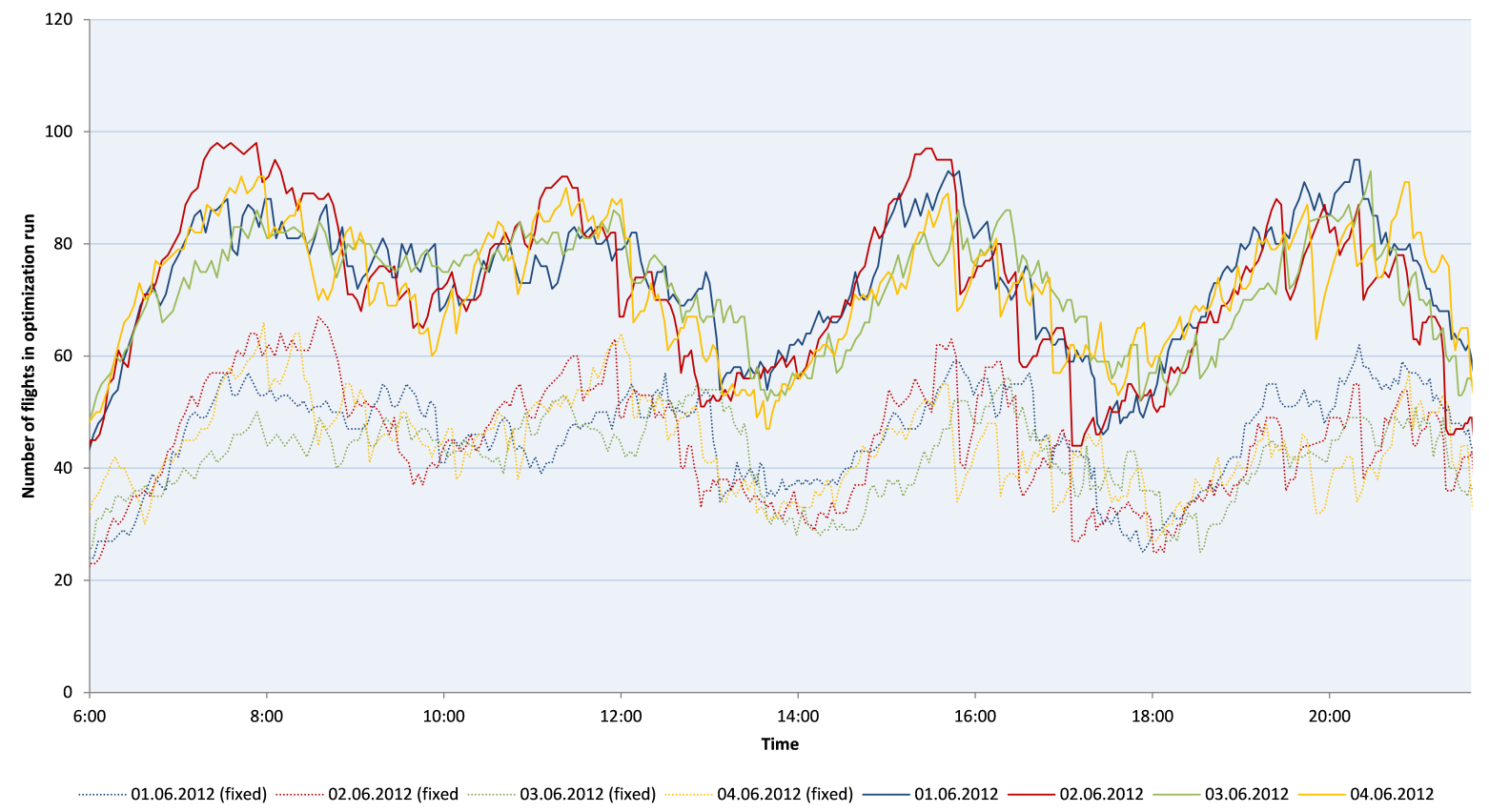

Figure 3: Number of flights in the model during different days. The upper solid lines show all flights and the lower dotted lines the fixed flights. Colors refer to the dates, as indicated below the figure.

The model was implemented and gradually used as decision support tool for Frankfurt Airport. To choose the right decision parameters we did several simulation runs on real-world data from the past. The results presented in Section 4.2-4.3 are based on historic data from the 1st to the 4th of June (2012) and included the achieved quality values $q$.

Figure 3 shows the problem size in terms of number of flights during different days. There are several peaks which is typical for hub airports. Flights are considered as fixed if the dispatcher has made the final assignment or the flight has arrived at its parking position. Then no further possibilities are considered in the optimization runs. The number of fixed flights depends on the arrival of the aircraft and the time of the final decision of the dispatcher.

Figure 4 depicts the number of generated assignments during the studied days. In our case study there were five baggage claim halls in use and in total 35 baggage carousels available. The figures show that not all possible assignments are generated. The number of the feasible assignments is much smaller than the theoretical number (number of flights multiplied with the number of baggage carousels) since there are many limitations to baggage claim halls due to the infrastructure and also a large part of the flights is already fixed.

Decision quality is in the following measured by comparing the results of a studied strategy with the global best solution as described in Section 3.3. While studying a specific strategy we assume that only the data which were known in reality at a specific point in time are used when calculating the decisions at this point in time. The global best solution can be determined with the help of all known data. Furthermore, quality $q$ of the model is measured by the comparison 


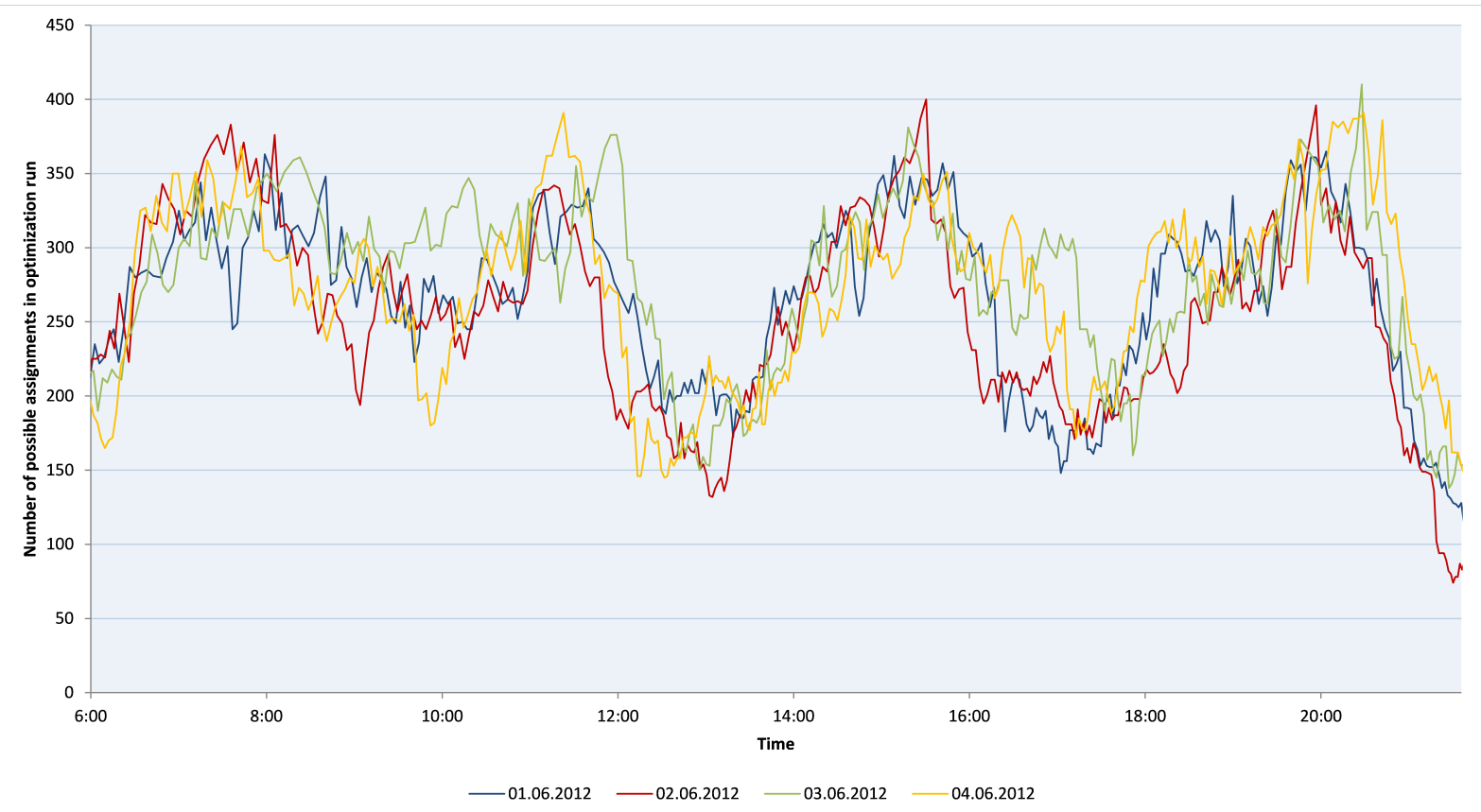

Figure 4: Number of possible assignments in the model during different days. Colors refer to the dates, as indicated below the figure.

with global best solution. A completely robust solution strategy capturing all uncertainties would result in the global best solution with $q=1.0$.

\subsection{Model verification}

The model was implemented with .net C\#, IBM Ilog OPL and IBM Ilog Cplex 12.2. The runtime for solving the model is below one second in nearly all cases. In the setup at Frankfurt Airport, most of the time is spent for communication with the database and preparation of the data. Data preparation has shown to be an important issue since the solution quality depends on the quality of input data. The problem is that different data sources exist, and the data sources can be contradicting and unreliable.

The setup at Frankfurt Airport is as follows: One dispatcher is responsible for all decisions of all arriving flights. During peak times there can be several flights per minute. The dispatcher is supported with suggestions from the model and can accept or change the suggestions. All flights arriving within the next 60 minutes are considered. The model is solved on the most recent data at least every two minutes.

The decision support tool has gradually been implemented since 2011. The model has shown to be robust with respect to changes in business processes e.g. changes of airline preferences or infrastructure. Furthermore, all appearing extreme situations (e.g. peaks, bad weather with heavy delays) can be handled.

The dispatchers followed the suggestions from the model for about $80 \%$ of all flights on 
average. At some test days it was shown that almost $100 \%$ acceptance are possible with good operational results. Some changes are necessary because of the bad data quality from some flights. Since the strategic decision of the management is to give the final responsibility to the dispatchers and keep them involved in case of any unforeseen events they are encouraged to make their own decisions. The model should decrease workload, help in finding good solutions and in locating a start point for generating the final schedules.

In general it was observed that decision quality increased since fewer errors (e.g. wrong baggage claim hall) occurred and the dispatch strategy is harmonized over all dispatchers.

Since 2010 the passenger volume has increased by $33 \%$ at Frankfurt Airport. Additionally, the workload of the dispatcher was increased by a new runway and a large expansion of the terminal building. In connection with the Great to have you here initiative, Fraport in the recent years reported an increased passenger satisfaction and measured an improvement of the inbound baggage processes. Despite increased workload the better schedules were still achieved by one dispatcher responsible for assigning the baggage carousels. The introduced decision support by the presented model helped to achieve this goal.

Further, we conducted a theoretical study for a day with a low acceptance rate. We compared the decisions from the dispatcher against the decisions based on the model. At the selected day the dispatcher accepted $69 \%$ of the suggestions. The model was rerun for each data set of the day and the decisions were fixed at the final arrival of the aircraft. Comparing the objectives for the whole day shows an improvement of the overall objective of $13 \%$ bringing the quality $q$ substantial closer to 1.0. This shows that a theoretical improvement of the results according to the defined objective is possible if the the dispatcher follows the suggestions from the model more closely. Analyzing the different criteria shows that the focus of the dispatcher was limited to only a few criteria, like the airline preferences where his decisions showed better values. Especially, the criteria concerning the combination of flights showed substantial better results in the optimized case. There were fewer occurrences of simultaneous handling of several flights. Furthermore, we identified three flights where the dispatcher decided against given rules. Some of the observed differences can be explained by wrong data e.g. number of arrival bags or additional communication of the dispatcher with involved process partners e.g. airlines. A detailed study broken down to the individual objective terms which compares dispatcher decision versus model results is presented in the Technical Report [5].

\subsection{Time of decisions}

An important issue for the control of the decision process for the BCAP is the time of the final decision for each flight. Because of the changing data it is beneficial to decide as late as possible. On the other hand ground handlers have to prepare for the arriving flights and need the information about the handling location in due time. For balancing these conflicting needs we studied the impact of decision time on measured quality.

Figure 5 shows the development of decision quality $q$ for increasing gaps between the point in time of the decision and the actual handling. In these runs the optimization was fixed when the depicted flight status was reached. The findings show that the best results are achieved when 


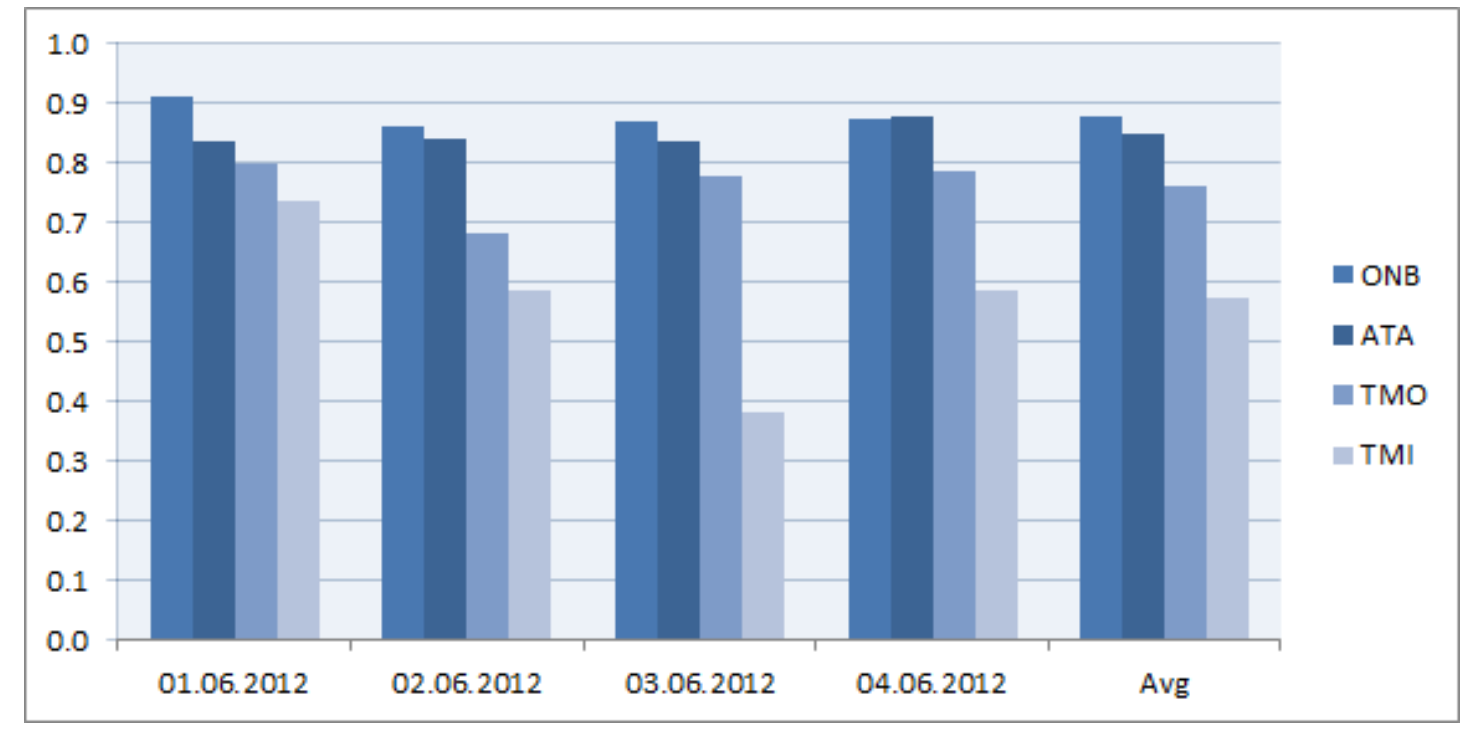

Figure 5: Decision quality as percentage of the global best solution. ONB: Arrival at parking position. ATA: Landing at airport. TMO: Ten minutes before estimated landing. TMI: Thirty minutes before estimated landing

the decision is not fixed until the flight has arrived at the airport (time status $\mathrm{ONB}^{1}$ or $\mathrm{ATA}^{2}$ ). In general the results when fixing the decisions at ONB are better compared to the fixation at ATA. For one day the ATA strategy showed better results than the ONB strategy. The reason was random effects caused by uncertainty since the arrival time at the handling facilities changed after ONB. The results before the arrival $\left(\mathrm{TMO}^{3}\right.$ and $\left.\mathrm{TMI}^{4}\right)$ at the airport are measurably worse than after the landing. Especially the results under the TMI strategy are $30 \%$ worse than the results measured with the ONB strategy.

The differences between the strategies are mainly due to the soft constraints. An earlier fixation of the decision variables causes simultaneous handling to occur more often, which shows that the robustness of the generated schedules is small.

\subsection{Time horizon of decisions}

Another important decision parameter is the look ahead time which is equal to the time horizon of the model. In this part of the case study we examine how solution quality is affected by the length of the time horizon of the model. The time horizon defines how many flights will be included in the model. We assume in this section that the decisions are fixed at ONB (see Section 4.2).

The results for different time horizons are shown in Figure 6. The results show that the

\footnotetext{
${ }^{1}$ arrival at parking position

${ }^{2}$ landing at airport

3 ten minutes before estimated landing

${ }^{4}$ thirty minutes before estimated landing
} 


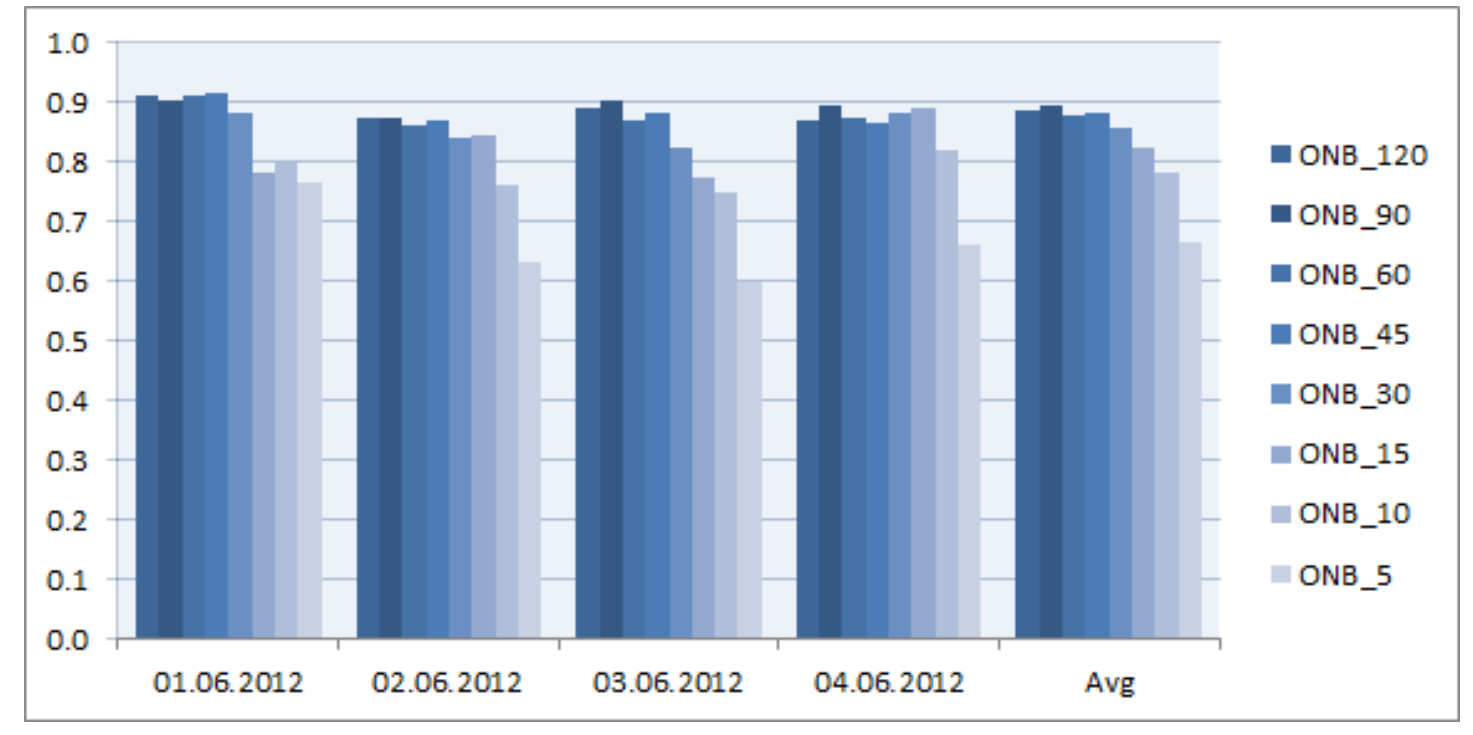

Figure 6: Decision quality in dependency of the time horizon. ONB_x means that all flights having an estimated arrival time within the next $\mathrm{x}$ minutes are considered by the model

longer time horizons (45 minutes and more) are all comparable. The reason is the large degree of uncertainty in the data. Increasing the time horizon further does not improve decision quality since it is likely that the arrival times of the flights are changing. Most remarkable is that even short planning horizons like a look ahead of 15 minutes still produces good results. In general we can see that an optimal decision horizon should be greater than or equal to 45 minutes. Extending the time horizon beyond 90 minutes does not improve solution quality. Further, the time used for data preparation increases with the length of the time horizon. This means that an upper bound of 90 minutes for the look ahead time is appropriate. The variations between the different strategies are mainly related to the soft constraints. Only for the runs with a short time horizon (5 and 10 minutes) also the direct costs (like airline preferences) were worse, compared to the runs with longer time horizons. In these cases, the model has much less freedom to assign the flights since most flights are already fixed. This is an important result since the dispatchers focused only on a small section of the current flights in the past. For example they looked only at flights between TMO and ATA at one time.

\subsection{Load variation}

In this section we study the behavior of the model when the problem size is increased. The load is defined in this context as number of flights which have to be scheduled in a certain time period. Furthermore, we show the impact on the airport infrastructure and process quality when the load is increased.

We selected the run with most flights of all test days. The peak was observed at 2nd of June around 7.30 AM. Starting with the results of the run at this point in time we increased the number of flights. Since all our results are based on real world data we cannot add randomly generated 
Table 5: Results of load variation

\begin{tabular}{lrrrrr} 
Run & $\begin{array}{r}\text { \# fixed } \\
\text { flights }\end{array}$ & $\begin{array}{r}\text { \# all } \\
\text { flights }\end{array}$ & \# assignments & $\begin{array}{l}\text { Solution } \\
\text { time }\end{array}$ & $\begin{array}{l}\text { Objective } \\
\text { value }\end{array}$ \\
\hline Original run & 40 & 98 & 501 & 20.02 & 0.3900 \\
Squeeze10 & 40 & 104 & 555 & 0.45 & 4.8509 \\
Squeeze20 & 40 & 107 & 588 & 3.42 & 2.7398 \\
Squeeze30 & 40 & 110 & 621 & 1.38 & -0.5070 \\
Squeeze40 & 40 & 114 & 662 & 11.88 & -2.6602 \\
Squeeze50 & 40 & 120 & 728 & 16.55 & -22.1660 \\
Squeeze50 additional bc & 40 & 120 & 896 & 21.91 & -2.8725 \\
Two days combined & 73 & 174 & 1124 & 12.25 & -14.0338 \\
\hline
\end{tabular}

flights. A flight involves many different features which are used in the model and need to fit together. Therefore we decided to use a "time squeezing" approach which means that we shrink time by a specified factor. For example squeezing by $10 \%$ with a time horizon of 60 minutes includes all flights within the next 66 minutes of the real world case. We chose to fix the decision at $\mathrm{ONB}$ and to limit the time horizon to 60 minutes.

Table 5 shows the number of flights and other important features of the generated test cases. In the first five cases we increase the number of flights in steps of $10 \%$. A squeezing of $50 \%$ means that we have to schedule the flights of the next 90 minutes within only one hour. Afterwards we tested the model adding 9 more baggage carousels, meaning an increase of resource availability of almost $20 \%$ (this run is named "Squeeze 50 additional bc"). Finally, we overlaid the flight data with the flights from the following day, almost doubling the number of flights (this run is named "Two days combined"). It is easy to see that the number of flights does not increase proportional to the squeezing factor. The reason is the typical peak structure of the traffic at an airport.

All test instances can be solved within a half a minute which confirms our experiences during the daily use of the model. We cannot observe a correlation of the size of instances and solution time. The results show that even an increased load by a factor of two will be solvable with a standard MIP solver. This leaves enough space for increasing traffic volume in the future. Today, there exist no airports with a traffic volume above the bound.

From the operational perspective it is interesting how the different objectives develop in these test instances. A direct comparison of the overall objective function is not possible since it depends on the number of considered flights. For instances with squeezing factor $50 \%$ we can see that adding additional resources produces better solutions but slightly increases solution time.

Looking at the different objective criteria we observe higher penalties for violation of the buffer and simultaneous handling constraints, when increasing the load. The criteria directly associated to an assignment like airline preferences are hardly affected by the increase in load. The increase of the load between the $40 \%$ to the $50 \%$ instance produces significantly worse results because the limits of the airport infrastructure are reached. A handling at this load level causes bad process quality since too many flights have to be handled simultaneously. This can be 
compensated by adding additional resources to the test instances. After increasing capacity about $20 \%$ (see run "Squeeze50 additional bc" in Table 5) a significant improvement of the results can be observed. The results do not reach the same quality as the instance with fewer flights. This is a consequence of more flight, due to longer transportation times becoming necessary for some flights in order to fit into the infrastructure.

The results show that the model is also suited to identify bottlenecks in the process and bounds of the infrastructure. Investigating the details of the results it is also possible to identify the specific problem area of the airport, like one of the baggage claim halls.

\subsection{Managerial insights}

The case study has shown that it is possible to enhance decision quality and thereby also process quality. The model enables a holistic approach taking several aspects into consideration. This leads to considerably better solutions than splitting the problem into sub problems. The model can either support the dispatcher or decide automatically. Furthermore, the results show that a human dispatcher focuses on the most important and obvious objective criteria but an IT supported decision tool can help in determining an improved way to balance all relevant criteria according to the objective.

In the past a dispatcher without support from the model considered a planning horizon of approximately ten minutes. He looked at all flights between TMO and ATA. The flights were fixed not later than ATA. The case study has shown that it is important to look at all flights arriving within the next 45 minutes. Furthermore, any fixation of the decision before ATA should be avoided. Even manual decision making can be improved if the decision processes are adapted according to these results. The decision of whether to use an automated model depends on the data quality. In general our experiments showed that additional support from the presented model will increase process quality.

For the time horizon of decisions we recommend 45 or 60 minutes. Planning further ahead can make sense if information is needed for staffing of the resources or the ground handlers but this will lead to an increased solution time because of increased data volume. The longer solution time is driven by the communication between the systems and the data preparation part.

A qualitative evaluation show that the quality $q$ of the solutions can be increased when the model is used compared to manual decision making. Dispatchers fix the flights early and look only a few minutes ahead. Considering many flights simultaneously and fixing flight late increases solution quality by reducing uncertainty.

A reduction of uncertainty could help in improving results. A better prognosis of the input data e.g. arrival times and number of bags will lead to a better process quality i.e. reliable unloading or transport service. Further studies can show the dependency of the degree of uncertainty and decision parameters.

Furthermore, even large instances can be solved directly, and it is not expected that an increasing traffic volume will cause problems. The model helps understanding the use of the infrastructure and can help to identifying bottlenecks in the process. 


\section{Conclusion}

We presented a general and flexible model which is easily extendible to changing business processes and objectives. It was proven that the granularity of the model is suited to support the daily decision process and operations and can also be used for strategic decisions and analysis. The advantage is that the results are easily comparable and it is not necessary to translate the results to different aggregation levels for strategic, tactical or operational planning. This is one of the major advantages of the presented model.

The presented results show that the introduced model is applicable for the BCAP and helps in improving process quality. The model combines the different needs of the different stake-holders and provides satisfying results for all involved parties. The application at Frankfurt Airport shows that the model in a satisfactory manner represents the real-world and is not only of theoretical use. It can be the basis for examination of different dispatch strategies and infrastructure analysis.

The results of the case study show that the final decision for the single flights should be between ONB and ATA. Furthermore, the time horizon of the considered flights should be between 45 and 90 minutes. The possible uses of the model are manifold. For example it can also be used to determine a bound on the minimum available capacity in dependency of a wished quality level. Another example is to study the effects of capacity reductions.

The presented research depends upon results of other research areas. Examples are the determination of a forecast of a data arrival profile of the passengers or the prognosis of the number of bags on the different flights. Furthermore, the stochasticity of the arrival of the trips is an important topic which can be studied. The impact of the soft factors allows for integrating the model in daily operations, which is another important point.

Finally, we work on a framework for solving the decision problems of baggage handling and staff / task scheduling in an integrated way. The aim of the approach is a better utilization of the staff and to overcome the bottlenecks between the different processes resulting in a better overall quality.

\subsection{Possible extensions}

The presented model has a flexible design, and allows for extensions of constraints or objectives. By adding additional soft constraints it is possible to include further aspects of baggage handling. For example the avoidance of bottlenecks in the processes influenced by the selected baggage carousel can be modeled as an additional soft constraint. Examples of such processes are the passenger flow in the terminal building, the capacities of the bus arrivals or passenger arrival at border control.

Another extension implemented at Frankfurt Airport is to allow the selection of more than one baggage carousel per flight. In this case an assignment will use resources of two baggage carousels simultaneously. It is easy to see that this requires only a minor change of the model involving the parameter $u_{a, r, t}$. This extension may for instance be used for separate handling of bags from priority passengers or the crew and passengers traveling in economy class.

It can be useful to apply different sets of timeslots for calculating the soft constraints. For some capacity constraints it does not make sense to calculate a balanced distribution for small 
timeslots. In this case, larger timeslots can be introduced to calculate these capacity constraints. Furthermore, rolling intervals can help in avoiding unstable results due to the artificial bounds between the timeslots. Rolling interval means that the resource utilization is calculated for different sets of timeslots. The sets of timeslots differ in their shifted start points.

Another option is to allow delayed baggage handling for specific flights. This can help avoiding peaks in handling and to find a better balanced solution. At most airports this idea means an adoption of the existing handling processes and difficulties in the operational processes can occur.

For increasing decision quality $q$ it can be an option to include data from flights outside the defined time horizon of the data. These flights should not be displayed to the dispatcher and there is no bonus if they are assigned to the same baggage carousel in two consecutive optimization runs. This approach can help in avoiding instability by the appearance of new flights which are in conflict with already planned flights.

\section{Conflict of interest}

On behalf of all authors, the corresponding author states that there is no conflict of interest.

\section{References}

[1] A Abdelghany, K Abdelghany, and R Narasimhan. Scheduling baggage-handling facilities in congested airports. Journal of Air Transport Management, 12(2):76-81, 2006.

[2] Fraport AG. Baggage management and infrastructure. Web, 2012.

[3] G Andreatta, L Brunetta, and L Righi. Evaluating terminal management performances using slam: The case of athens international airport. computers. Computers \& Operations Research, 34:1532-1550, 2007.

[4] A Ascó, JAD Atkin, and EK Burke. The airport baggage sorting station allocation problem. In Proceedings of the 5th Multidisciplinary International Scheduling Conference, pages 419-444, 2011.

[5] T Barth. Optimal assignment of incoming flights to baggage carousels at airports. Technical Report Report 5, DTU Management Engineering, 2013.

[6] T Barth and M. Franz. Gemischt-ganzzahlige Optimierung in Echtzeit am Beispiel der Steuerung des Transfergepäckumschlags am Frankfurter Flughafen (In German). In Dirk Mattfeld, editor, Gemeinsame Fachtagung der Gesellschaft für Operations Research und der Deutschen Gesellschaft für System Dynamics, pages 38-66. Braunschweig: Institut für Wirtschaftsinformatik, 2008.

[7] T Barth and D Pisinger. Scheduling of outbound luggage handling at airports. In D. Klatte, H.-J. Lüthi, and K. Schmedders, editors, Operations Research Proceedings 2011, pages 251-256. Springer, 2012. 
[8] F Delonge. Balancing load distribution on baggage belts at airports. In Operations Research Proceedings, International Annual Conference of the German Operations Research Society in Hannover, pages 499-505. Springer, 2012.

[9] G Diepen, JM Van Den Akker, JA a Hoogeveen, and JW Smeltink. Finding a robust assignment of flights to gates at amsterdam airport schiphol. Journal of Scheduling, 15(6):703$715,2012$.

[10] M Frey, C Artigues, R Kolisch, and P Lopez. Scheduling and planning the outbound baggage process at international airports. In 2010 IEEE International Conference on Industrial Engineering and Engineering Management, pages 2129-2133, 2010.

[11] M Frey, F Kiermaier, and R Kolisch. Optimizing inbound baggage handling at airports. Transportation Science, 51(4):1210-1225, 2017.

[12] A Ghobrial, CF Daganzo, and T Kazimi. Baggage claim area congestion at airports: an empirical model of mechanized claim device performance. Transportation Science, 16(2):246-260, 1982.

[13] E Huang, I Liu, and JT Lin. Robust model for the assignment of outgoing flights on airport baggage unloading areas. Transportation Research Part E: Logistics and Transportation Review, 115:110-125, 2018.

[14] E Huang, P Mital, M Goetschalckx, and K Wu. Optimal assignment of airport baggage unloading zones to outgoing flights. Transportation Research Part E: Logistics and Transportation Review, 94:110-122, 2016.

[15] C Malandri, M Briccoli, L Mantecchini, and F Paganelli. A discrete event simulation model for inbound baggage handling. Transportation Research Procedia, 35:295-304, 2018.

[16] DW Pentico. Assignment problems: A golden anniversary survey. European Journal of Operational Research, 176(2):774-793, 2007.

[17] D Pisinger and SH Rude. Advanced algorithms for improved baggage handling. Journal of Airport Management, 14, 2020.

[18] D Pisinger and R Scatamacchia. The baggage belt assignment problem, 2020. arXiv preprint arXiv:2006.03365.

[19] SITA. Air transport industry insights, the passenger IT trend survey 2016. https://www.sita.aero/resources/type/surveys-reports/passenger-it-trends-survey-2016, 2016. (accessed 14th April, 2020).

[20] M Tamiz, D Jones, and C Romero. Goal programming for decision making: An overview of the current state-of-the-art. European Journal of Operational Research, 111(3):569-581, 1998. 
[21] V Tošić. A review of airport passenger terminal operations analysis and modelling. Transportation Research Part A: Policy and Practice, 26(1):3-26, 1992.

[22] J Wang, J Cao, S Wang, Z Yao, and W Li. Irda: Incremental reinforcement learning for dynamic resource allocation. IEEE Transactions on Big Data, 2020. 


\section{A Notation}

All parameters and variables are defined in the main text, but for ease of reference, an overview is given in Tables 6-8.

Table 6: List of indices and sets

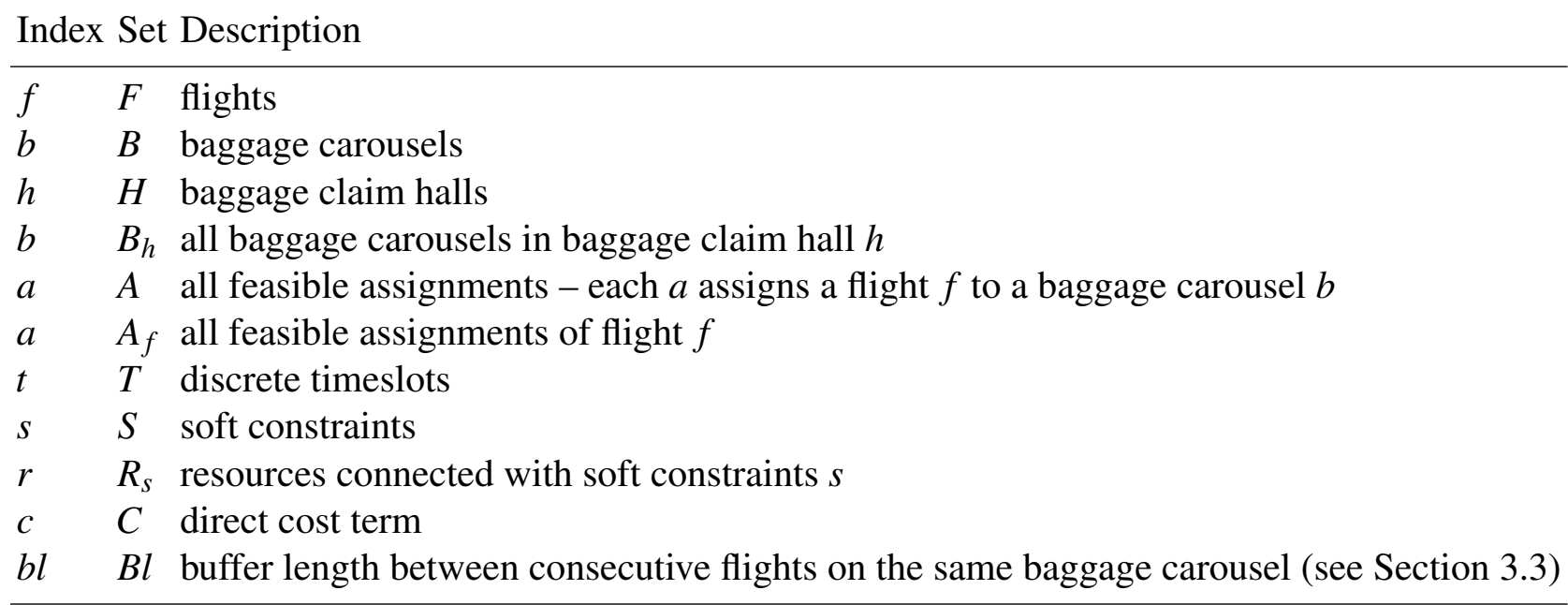

Table 7: List of parameters

\begin{tabular}{ll}
\hline Parameter & Description \\
\hline$l$ & length of timeslots \\
$L$ & planning horizon: $L=|T| \cdot l$ \\
$u_{a, r, t}$ & use of resource $r$ by assignment $a$ in timeslot $t$ \\
$u_{a, b, t, b l}$ & use of baggage carousel $b$ by assignment $a$ in timeslot $t$ when buffer length $b l$ is \\
$C A P_{r, t}$ & applied (see Section 3.3 ) \\
$n_{c}$ & capacity of resource $r$ at timeslot $t$ \\
$w_{c}$ & indicates minimization $\left(n_{c}=1\right.$ ) or maximization $n_{c}=-1$ of cost term $c$ \\
$w_{s}$ & weight of soft constraint $s$ \\
$w^{B H}$ & weight of balanced use of baggage claim halls \\
$w^{B C}$ & weight of balanced use of baggage carousels \\
$w_{b l}$ & weight of buffer constraint with buffer length $b l$ \\
$o_{a, c}$ & cost of assignment $a$ and cost term $c$
\end{tabular}


Table 8: List of variables

\begin{tabular}{|c|c|}
\hline Variable & Description \\
\hline$x_{a}$ & indicates if assignment $a$ is selected \\
\hline$y_{r, t}$ & measures the violation of the capacity constraint defined for resource $r$ at timeslot $t$ \\
\hline$y_{b, t, b l}$ & $\begin{array}{l}\text { measures the violation of the buffer constraint defined for baggage carousel } b \text { at } \\
\text { timeslot } t \text { when buffer length } b l \text { is applied (see Section 3.3) }\end{array}$ \\
\hline$y^{B H, \text { mean }}$ & average use of all baggage claim halls $H$ \\
\hline$y_{h}^{B H,+}$ & $\begin{array}{l}\text { measures the positive deviation of the use of baggage claim hall } h \text { of the average } \\
\text { use } y^{B H, \text { mean }}\end{array}$ \\
\hline$y_{h}^{B H,-}$ & $\begin{array}{l}\text { measures the negative deviation of the use of baggage claim hall } h \text { of the average } \\
\text { use } y^{B H, \text { mean }}\end{array}$ \\
\hline$y_{h}^{B C, \max }$ & measures the maximum use of a baggage carousel in baggage claim hall $h$ \\
\hline$y_{h}^{\ddot{B C}, \min }$ & measures the minimum use of a baggage carousel in baggage claim hall $h$ \\
\hline$y_{h}^{B C}$ & measures distance between $y_{h}^{B C, \max }$ and $y_{h}^{B C, \min }$ for baggage claim hall $h$ \\
\hline
\end{tabular}




\section{B Complete model}

For the sake of completeness we here present the complete model as described in the paper:

$$
\begin{aligned}
& \min \sum_{a \in A, c \in C} n_{c} \cdot w_{c} \cdot o_{a, c} \cdot x_{a}+\sum_{s \in S} w_{s} \sum_{r \in R_{s}, t \in T} y_{r, t}+w^{B H} \sum_{h \in H} y_{h}^{B H}+w^{B C} \sum_{h \in H} y_{h}^{B C} \\
& \text { s.t. } \sum_{a \in A_{f}} x_{a}=1 \quad \forall f \in F \\
& \sum_{a \in A} u_{a, r, t} \cdot x_{a} \leq C A P_{r, t}+y_{r, t} \quad \forall r \in R_{s}, t \in T \\
& y^{B H, \text { mean }}=\frac{\sum_{a \in A, h \in H, t \in T} u_{a, h, t} \cdot x_{a}}{\sum_{h \in H, t \in T} C A P_{h, t}} \\
& y^{B H, \text { mean }}+y_{h}^{B H,+}-y_{h}^{B H,-}=\frac{\sum_{a \in A, t \in T} u_{a, h, t} \cdot x_{a}}{\sum_{t \in T} C A P_{h, t}} \quad \forall h \in H \\
& y_{h}^{B H}=y_{h}^{B H,+}+y_{h}^{B H,-} \quad \forall h \in H \\
& y_{h}^{B C, \max } \geq \frac{\sum_{a \in A, t \in T} u_{a, b, t} \cdot x_{a}}{\sum_{t \in T} C A P_{b, t}} \quad \forall h \in H, b \in B_{h} \\
& y_{h}^{B C, \text { min }} \leq \frac{\sum_{a \in A, t \in T} u_{a, b, t} \cdot x_{a}}{\sum_{t \in T} C A P_{b, t}} \quad \forall h \in H, b \in B_{h} \\
& y_{h}^{B C}=y_{h}^{B C, \text { max }}-y_{h}^{B C, \text { min }} \quad \forall h \in H \\
& \sum_{a \in A} u_{a, b, t, b l} \cdot x_{a} \leq 1+y_{b, t, b l} \quad \forall h \in H, b \in B_{h}, t \in T, b l \in B l \\
& y^{B H, \text { mean }} \geq 0 \\
& y_{h}^{B H} \geq 0, \quad y_{h}^{B H,+} \geq 0, \quad y_{h}^{B H,-} \geq 0 \quad \forall h \in H \\
& y_{h}^{B C} \geq 0, \quad y_{h}^{B C, \max } \geq 0, \quad y_{h}^{B C, \text { min }} \geq 0 \quad \forall h \in H \\
& x_{a} \in\{0,1\} \quad \forall a \in A
\end{aligned}
$$

